\title{
Development and Validation of a Nomogram Model to Predict Acute Kidney Disease After Nephrectomy in Patients with Renal Cell Carcinoma
}

This article was published in the following Dove Press journal:

Cancer Management and Research

\author{
Xiao-Ying $\mathrm{Hu}^{1-5}$ \\ Dong-Wei Liu ${ }^{1-5}$ \\ Ying-Jin Qiao ${ }^{1-5}$ \\ Xuan Zheng ${ }^{6}{ }^{6}$ \\ Jia-Yu Duan ${ }^{1-5}$ \\ Shao-Kang Pan ${ }^{1-5}$ \\ Zhang-Sou Liu ${ }^{1-5}$
}

'Department of Nephrology, The First Affiliated Hospital of Zhengzhou University, Zhengzhou 450052, People's Republic of China; ${ }^{2}$ Research Institute of Nephrology, Zhengzhou University, Zhengzhou 450052, People's Republic of China; ${ }^{3}$ Research Center for Kidney Disease, Zhengzhou 450052, Henan Province, People's Republic of China; ${ }^{4}$ Key Laboratory of Precision Diagnosis and Treatment for Chronic Kidney Disease in Henan Province, Zhengzhou 450052, People's Republic of China; ${ }^{5}$ Core Unit of National Clinical Medical Research Center of Kidney Disease, Zhengzhou 450052, People's Republic of China; ${ }^{6}$ Department of Radiation Oncology, National Cancer Center/ Cancer Hospital, Chinese Academy of Medical Sciences (CAMS) and Peking Union Medical College (PUMC), Beijing, I0002I, People's Republic of China

Correspondence: Zhang-Sou Liu Department of Nephrology, The First Affiliated Hospital of Zhengzhou University, Zhengzhou 450052, Henan Province, People's Republic of China Tel +86-037I-6629592I

Fax +86-037I-66970906

Email zhangsuoliu@zzu.edu.cn
Purpose: To develop and validate a nomogram model to predict the occurrence of acute kidney disease (AKD) after nephrectomy.

Patients and Methods: A retrospective cohort including 378 patients with renal cell carcinoma (RCC) who had undergone radical or partial nephrectomy between March 2013 and December 2017 at the First Affiliated Hospital of Zhengzhou University was analyzed. Of these, patients who had undergone surgery in an earlier period of time formed the training cohort ( $\mathrm{n}=265$ ) for nomogram development, and those who had undergone surgery thereafter formed the validation cohort $(n=113)$ to confirm the model's performance. The incidence rate of $\mathrm{AKD}$ was measured. Univariate and multivariate logistics regression analysis was used to estimate the independent risk factors associated with AKD. The independent risk factors were incorporated into the nomogram. The accuracy and utility of the nomogram were evaluated by calibration curve and decision curve analysis, respectively.

Results: Overall, AKD occurred in $27.5 \%$ and $28.3 \%$ of patients in the training and validation cohorts, separately. The final nomogram included surgery approach, Charlson comorbidity index (CCI), and the decrement of eGFR. This model achieved good concordance indexes of $0.78(95 \% \mathrm{CI}=0.71-0.84)$ and $0.76(95 \% \mathrm{CI}=0.67-0.86)$ in the training and validation cohorts, respectively. The calibration curves and decision curve analysis (DCA) demonstrated the accuracy and the clinical usefulness of the proposed nomogram, separately. Conclusion: The nomogram accurately predicts AKD after nephrectomy in patients with RCC. The risk for patients' progress into AKD can be determined, which is useful in guiding clinical decisions.

Keywords: kidney cancer, renal cell carcinoma, nephrectomy, acute kidney disease, nomogram

\section{Introduction}

Kidney cancer has become the 9th most common cancer among men and the 14th most common cancer among women globally. ${ }^{1} \mathrm{RCC}$ is the most common pathology subtype of kidney cancer. ${ }^{2}$ Surgery is still the optimal treatment approach for RCC according to the European Association of Urology Guidelines. ${ }^{3}$ Patients who are able to undergo nephrectomy are usually long-term cancer survivors. ${ }^{4}$ However, decreased kidney function or contralateral kidney failure are common after nephrectomy. Previous studies demonstrated that renal function reduction occurred 
in $25-40 \%$ patients postoperatively. ${ }^{5-8}$ Besides, other studies have shown that the reduction in renal function was correlated with decreased life quality and increased mortality rate. ${ }^{9,10}$ Thus, it is of vital importance to identify the risk factors for renal function reduction and accurately predict renal function impairment after nephrectomy in order to provide timely intervention.

In clinical practice, acute kidney injury (AKI) and chronic kidney disease (CKD) are the most common outcomes of renal function reduction after surgery. AKI is defined as a $50 \%$ increase in serum creatinine ( $\mathrm{SCr}$ ) within 7 days after surgery or a $0.3 \mathrm{mg} / \mathrm{dL} \mathrm{SCr}$ increase within 48 hours according to the Kidney Disease: Improving Global Outcomes (KDIGO) clinical practice guidelines. ${ }^{11}$ Alteration in kidney function and structure for over 3 months are defined as CKD. ${ }^{12,13}$ However, more and more evidence has demonstrated that $\mathrm{AKI}$ and $\mathrm{CKD}$ are strongly connected and patients with AKI have an increased risk of developing CKD. ${ }^{14}$ Thus, the term acute kidney disease (AKD) has been put forward by KDIGO guidelines in order to define the course of disease after AKI in patients in whom the renal pathophysiologic processes are still ongoing. ${ }^{13}$ During the time window of AKD, interventions such as patient education, drug adjustment, and frequent follow-up can be initiated and may reverse the process of the disease. ${ }^{13}$ Thus, it is important to identify the risk factors of $\mathrm{AKD}$ and patients with high risk of developing AKD in order to provide timely intervention. Previous studies have demonstrated that risk factors such as surgery approach, perioperative eGFR, complications, age, and sex were associated with AKI and CKD postoperatively. ${ }^{15,16}$ However, as far as we know, the incidence rate and risk factors for $\mathrm{AKD}$ have not been studied and the prediction model for AKD after nephrectomy in RCC patients has not been established.

Therefore, we retrospectively analyzed the incidence rate of postoperative AKD in RCC patients and risk factors associated with AKD. Besides, we developed a nomogram to predict the occurrence of AKD in the early stage after surgery among the patients with RCC.

\section{Patients and Methods}

\section{Patients}

During March 5, 2013 and December 31, 2017, patients who had undergone nephrectomy for histologically confirmed RCC were retrospectively collected at the First Affiliated Hospital of Zhengzhou University. The eligibility criteria for the study were as follows: I) patients older than 18 years; II) patients who had undergone radical or partial nephrectomy for kidney cancer for the first time; and III) patients with available data of baseline $\mathrm{SCr}$ and SCr level within 3 months after surgery. Exclusion criteria included postoperative follow-up time less than 3 months, incomplete data, or any previous kidney surgery (ipsilateral or contralateral). Patients $(70 \%)$ included in the training cohort were treated with surgery between March 5, 2013 and March 6, 2017. Patients (30\%) who had undergone surgery between March 6, 2017 and December 21, 2017 entered the validation cohort.

\section{Clinicopathologic Characteristics and Predictors}

The occurrence of AKD after surgery was the end point of the study, which was defined as AKI, eGFR $<60 \mathrm{~mL} / \mathrm{min} /$ $1.73 \mathrm{~m}^{2}$ for less than 3 months, a $\geq 35 \%$ decrease in eGFR or a $>50 \%$ increase in $\mathrm{SCr}$ for less than 3 months according to the criteria recommended by the Acute Disease Quality Initiative (ADQI) 16 workgroup. ${ }^{13}$ AKI was defined as a $50 \% \mathrm{SCr}$ increase within 7 days after surgery or a $0.3 \mathrm{mg} / \mathrm{dL} \mathrm{SCr}$ increase within 48 hours according to the Kidney Disease: Improving Global Outcome (KDIGO) Clinical Practice Guideline for Acute Kidney Injury. ${ }^{17}$ Baseline SCr was defined as the latest measured $\mathrm{SCr}$ within 30 days preoperatively. The highest $\mathrm{SCr}$ level within 7 days after surgery was used to measure AKI. In addition, the highest SCr levels within 3 months postoperatively were recorded to evaluated the occurrence of AKD.

eGFR was calculated using the Chronic Kidney Disease Epidemiology Collaboration (CKD-EPI) formula. We used the baseline eGFR and eGFR within 7 days after surgery to calculate the decrement of eGFR, and the decrement is 0 when postoperative eGFR increased. Charlson comorbidity index (CCI) is a commonly used index to assess patients' burden of comorbidities and it was reported to be associated with adverse renal outcomes. ${ }^{18-20}$ The value is calculated based on 19 comorbid conditions as the comorbidities are incorporated into the score. The surgery approach in this study includes partial and radical nephrectomy. CT and ultrasound were used to confirm distant metastasis and venous tumor thrombus.

\section{Statistical Analysis}

Continuous variables are shown as mean (SD) and compared using an unpaired, 2-tailed $t$-test or Mann-Whitney 
test. Categorical variables between two cohorts were compared using the $X^{2}$ test or Fisher exact test. The univariate logistics regression was used to evaluate the risk factors for the occurrence of AKD in the training cohort. Variables with a $P$-value less than 0.1 in univariate logistics regression were involved in the multivariate logistics regression. A nomogram was formulated based on the independent risk factors of the multivariate logistic regression analysis. The calibration curve of the training cohort and validation cohort were generated to determine the deviation from the model's predicted probabilities, which was measured with 1,000 bootstrap samples to decrease the overfit bias. The clinical utility of the model was assessed by DCA. Data analysis was performed using SPSS v.22 (IBM Corporation, USA) and R version 3.6.2 (http://www.r-pro ject.org/).

\section{Results}

\section{Clinicopathologic Characteristics of Patients}

Our study included 378 RCC patients who have undergone nephrectomy. Patients were divided into training $(n=265)$ and validation $(n=113)$ cohorts according to the date of surgery. AKD occurred in 73 (27.5\%) and 32 $(28.3 \%)$ patients in the training and validation cohort, respectively. The baseline characteristics are summarized in Table 1 and Supplement Table 1. Most variables were similar between two cohorts; however, the surgery approach and operating time were statistically different between the two cohorts.

\section{Univariate and Multivariate Analysis of Risk Factors for AKD}

Variables in Table 1 were incorporated in univariate logistics regression analysis. Table 2 shows the result of univariate logistics regression analysis of 265 patients in the training cohort. In univariate logistics regression analysis, weight $(\mathrm{OR}=1.03,95 \% \mathrm{CI}=1.00-1.05, P=0.026)$, surgery approach (radical vs partial, $\mathrm{OR}=4.18,95 \% \mathrm{CI}=1.81-9.66$, $P=0.001)$, and decrement of eGFR $(\mathrm{OR}=1.06,95 \%$ $\mathrm{CI}=1.04-1.08, P<0.001)$ were statistically significant in predicting AKD. And then, variables with a $P$-value less than 0.1 in univariate logistics regression analysis were further analyzed with multivariate logistics regression. The results of multivariate logistics regression analysis are summarized in Table 3, among which the surgery approach
Table I Baseline Characteristics of the Patients in the Training Cohort and the Validation Cohort

\begin{tabular}{|c|c|c|c|}
\hline \multirow[t]{3}{*}{ Variables } & \multicolumn{2}{|l|}{ Cohort } & \multirow[t]{3}{*}{$P$-value } \\
\hline & Training & Validation & \\
\hline & $\mathrm{N}=265$ (\%) & $N=113$ (\%) & \\
\hline \multicolumn{4}{|l|}{ Age, years } \\
\hline$\leq 60$ & $177(66.8)$ & $73(64.6)$ & 0.680 \\
\hline$>60$ & $88(33.2)$ & $40(35.4)$ & \\
\hline \multicolumn{4}{|l|}{ Gender } \\
\hline Male & $168(63.4)$ & $81(71.7)$ & 0.120 \\
\hline Female & $97(36.6)$ & $32(28.3)$ & \\
\hline Weight, mean (SD), kg & $68.9(11.5)$ & $70.3(11.8)$ & 0.252 \\
\hline \multicolumn{4}{|l|}{$\mathrm{CCl}$} \\
\hline$<4$ & $237(89.4)$ & $98(86.7)$ & 0.448 \\
\hline$\geq 4$ & $28(10.6)$ & $15(13.3)$ & \\
\hline \multicolumn{4}{|l|}{ Surgery approach } \\
\hline Radical & $66(24.9)$ & $47(41.6)$ & \\
\hline Partial & $199(75.1)$ & $66(58.4)$ & 0.001 \\
\hline $\begin{array}{l}\text { Preoperative serum creatinine, } \\
\text { mean (SD), } \mu \mathrm{mol} / \mathrm{L}\end{array}$ & $76.7(55.5)$ & $73.9(21.2)$ & 0.641 \\
\hline \multicolumn{4}{|l|}{ Preoperative eGFR, } \\
\hline \multicolumn{4}{|l|}{$\mathrm{mL} / \mathrm{min} / 1.73 \mathrm{~m}^{2}$} \\
\hline$\geq 90$ & $177(66.8)$ & $73(64.6)$ & \\
\hline $60-89$ & $74(27.9)$ & $33(29.2)$ & 0.895 \\
\hline$<60$ & $14(5.3)$ & $7(6.2)$ & \\
\hline $\begin{array}{l}\text { Decrement of eGFR mean } \\
(\mathrm{SD}), \mathrm{mL} / \mathrm{min} / 1.73 \mathrm{~m}^{2}\end{array}$ & $25.3(17.1)$ & $22.8(15.4)$ & 0.164 \\
\hline \multicolumn{4}{|l|}{ Operating time, hours } \\
\hline$\leq 2$ & $133(50.2)$ & $33(29.2)$ & $<0.001$ \\
\hline$>2$ & $132(49.8)$ & $80(70.8)$ & \\
\hline \multicolumn{4}{|l|}{ Blood loss, $\mathrm{mL}$} \\
\hline$<150$ & $190(7 \mid .7)$ & $85(75.2)$ & 0.481 \\
\hline$\geq 150$ & $75(28.3)$ & $28(24.8)$ & \\
\hline \multicolumn{4}{|l|}{ Intraoperative minimum } \\
\hline \multicolumn{4}{|l|}{$\mathrm{SBP}, \mathrm{mmHg}$} \\
\hline$>90$ & $86(32.5)$ & $33(29.2)$ & 0.533 \\
\hline$\leq 90$ & $179(67.5)$ & $80(70.8)$ & \\
\hline $\begin{array}{l}\text { Time of SBP below } 90 \mathrm{mmHg} \text { in } \\
\text { surgery, mean (SD), minutes }\end{array}$ & $12.7(20.1)$ & $9.15(16.8)$ & 0.112 \\
\hline $\begin{array}{l}\text { Intraoperative urinary volume, } \\
\text { mean (SD), } \mathrm{mL}\end{array}$ & $246.0(193.0)$ & $\begin{array}{l}269.2 \\
(216.1)\end{array}$ & 0.285 \\
\hline Intraoperative fluid intake volume, & $1,638.9$ & I,734.3 & 0.200 \\
\hline mean (SD), $\mathrm{mL}$ & $(969.0)$ & $(894.9)$ & \\
\hline \multicolumn{4}{|l|}{ Pathological type } \\
\hline Clear cell & $204(77.0)$ & $92(81.4)$ & 0.096 \\
\hline Papillary cell & $9(3.4)$ & $8(7.1)$ & \\
\hline Chromophobe renal cell & $13(4.9)$ & $5(4.4)$ & \\
\hline Others & $39(14.7)$ & $8(7.1)$ & \\
\hline
\end{tabular}

(Continued) 
Table I (Continued).

\begin{tabular}{|c|c|c|c|}
\hline \multirow{3}{*}{ Variables } & \multicolumn{2}{|l|}{ Cohort } & \multirow{3}{*}{$P$-value } \\
\hline & Training & Validation & \\
\hline & $\mathrm{N}=265(\%)$ & $N=113(\%)$ & \\
\hline \multicolumn{4}{|l|}{ Tumor number } \\
\hline Solitary & $253(95.5)$ & $111(98.2)$ & 0.246 \\
\hline Multiple & $12(4.5)$ & $2(1.8)$ & \\
\hline \multicolumn{4}{|l|}{ Renal capsule invasion } \\
\hline No & $249(94.0)$ & III (98.2) & 0.074 \\
\hline Yes & $16(6.0)$ & $2(1.8)$ & \\
\hline \multicolumn{4}{|l|}{ Venous tumor thrombus } \\
\hline No & $248(93.6)$ & $107(94.7)$ & 0.681 \\
\hline Yes & $17(6.4)$ & $6(5.3)$ & \\
\hline \multicolumn{4}{|l|}{ TNM stage } \\
\hline I-II & $215(81.1)$ & $97(85.8)$ & 0.270 \\
\hline III-IV & $50(18.9)$ & $16(14.2)$ & \\
\hline
\end{tabular}

Notes: Continuous variables are displayed as median (standard deviation); Categorical variables are displayed as number (percentage).

Abbreviations: SD, standard deviation; $\mathrm{CCl}$, Charlson comorbidity index; eGFR, estimated glomerular filtration rate; SBP, systolic blood pressure.

$(\mathrm{OR}=3.47,95 \% \mathrm{CI}=1.40-8.59, P=0.007), \mathrm{CCI}(\mathrm{OR}=4.75$, 95\% CI $=1.75-12.87, P=0.002)$, and the decrement of eGFR (OR=1.06, 95\% CI=1.03-1.08, $P<0.001)$ were independent risk factors of AKD after nephrectomy.

Table 2 Univariate Logistic Regression Analysis of AKD Based on Perioperative Data in the Training Cohort

\begin{tabular}{|c|c|c|}
\hline Variables & OR $(95 \% \mathrm{CI})$ & $P$-value \\
\hline Age, $>60$ vs $\leq 60$, years & $\mathrm{I} .37(0.78-2.40)$ & 0.273 \\
\hline Gender, male vs female & $0.73(0.4 I-1.30)$ & 0.289 \\
\hline Weight, kg & $1.03(1.00-1.05)$ & 0.026 \\
\hline $\mathrm{CCl}, \geq 4$ vs $<4$ & $2.16(0.97-4.83)$ & 0.060 \\
\hline Surgery approach, radical vs partial & $4.18(1.81-9.66)$ & 0.001 \\
\hline Preoperative serum creatinine, $\mu \mathrm{mol} / \mathrm{L}$ & $1.00(1.00-1.01)$ & 0.546 \\
\hline \multicolumn{3}{|l|}{ Preoperative eGFR, $\mathrm{mL} / \mathrm{min} / 1.73 \mathrm{~m}^{2}$} \\
\hline $60-89$ vs $\geq 90$ & $0.92(0.50-1.68)$ & 0.775 \\
\hline$<60$ vs $\geq 90$ & $0.41(0.09-1.91)$ & 0.256 \\
\hline Decrement of eGFR, $\mathrm{mL} / \mathrm{min} / 1.73 \mathrm{~m}^{2}$ & $1.06(1.04-1.08)$ & $<0.001$ \\
\hline Operating time, $>2$ vs $\leq 2$ hours & $0.84(0.49-1.44)$ & 0.516 \\
\hline Blood loss, $\geq 150$ vs $<150, \mathrm{~mL}$ & $0.57(0.30-1.08)$ & 0.086 \\
\hline Intraoperative minimum SBP, $\leq 90$ vs $>90, \mathrm{mmHg}$ & $1.07(0.60-1.90)$ & 0.839 \\
\hline Time of SBP below $90 \mathrm{mmHg}$ in surgery, minutes & $1.00(0.99-1.01)$ & 0.497 \\
\hline \multicolumn{3}{|l|}{ Pathological type } \\
\hline Papillary cell vs clear cell & $0.29(0.04-2.39)$ & 0.252 \\
\hline Chromophobe renal cell vs clear cell & $0.70(0.19-2.65)$ & 0.602 \\
\hline Others vs clear cell & $0.61(0.26-1.39)$ & 0.237 \\
\hline Tumor number, multiple vs solitary & $2.78(0.87-8.91)$ & 0.086 \\
\hline Renal capsule invasion, yes vs no & $0.59(0.16-2.13)$ & 0.421 \\
\hline Venous tumor thrombus, yes vs no & $0.33(0.07-1.49)$ & 0.150 \\
\hline
\end{tabular}

Abbreviations: $\mathrm{OR}$, odds ratio; $\mathrm{Cl}$, confidence interval; $\mathrm{CCl}$, Charlson comorbidity index; eGFR, estimated glomerular filtration rate; SBP, systolic blood pressure.
Table 3 Multivariate Logistic Regression Analysis of AKD Based on Data in the Training Cohort

\begin{tabular}{|c|c|c|}
\hline Variables & OR (95\% Cl) & $P$-value \\
\hline Weight, kg & $1.02(0.99-1.05)$ & 0.122 \\
\hline Surgery approach, radical vs partial & $3.47(1.40-8.59)$ & 0.007 \\
\hline $\mathrm{CCl},<4$ vs $\geq 4$ & $4.75(1.75-12.87)$ & 0.002 \\
\hline Decrement of GFR, $\mathrm{mL} / \mathrm{min} / 1.73 \mathrm{~m}^{2}$ & $1.06(1.03-1.08)$ & $<0.001$ \\
\hline Blood loss, $\geq 150$ vs $<150, \mathrm{~mL}$ & $0.50(0.24-1.05)$ & 0.067 \\
\hline Tumor number, multiple vs solitary & $3.13(0.77-12.78)$ & 0.112 \\
\hline
\end{tabular}

Abbreviations: $\mathrm{OR}$, odds ratio; $\mathrm{Cl}$, confidence interval; $\mathrm{CCl}$, Charlson comorbidity index; eGFR, estimated glomerular filtration rate.

\section{Development and Validation of a Predictive Nomogram}

A nomogram model was constructed based on independent risk factors from multivariate logistics regression analysis for the prediction of postoperative AKD. Table 4 demonstrated the result of multivariate analysis of the variables included in the nomogram model. Figure 1 shows the nomogram which surgery approach, CCI, and the decrement of eGFR defined the individual risk of AKD in patients with RCC. In this nomogram, the decrement of eGFR is a continuous variable, every unit decrease in eGFR results in approximately a 1.1-point increase in risk points. CCI score $\geq 4$ and a radical surgery contributes 30 points and 22 points, respectively. Therefore, a patient with a $40 \mathrm{~mL} / \mathrm{min} / 1.73 \mathrm{~m}^{2}$ decrement in eGFR, CCI score $\geq 4$, and radical surgery has a $78 \%$ probability of developing AKD. Conversely, a patient with a $10 \mathrm{~mL} / \mathrm{min} / 1.73 \mathrm{~m}^{2}$ decrement in eGFR, CCI score $<4$, and palliative surgery has less than a $5 \%$ probability of developing AKD.

The nomogram demonstrated good accuracy in estimating the risk of AKD with a C-index of 0.78 (95\% $\mathrm{CI}=0.71-0.84)$ in the training cohort. The calibration curve in Figure 2A shows good concordance between the estimated risk of AKD and the actual presence of AKD. In addition, the probability of the occurrence of AKD in the validation cohort was calculated by the nomogram model. The nomogram of the validation cohort displayed a

Table 4 Multivariate Logistic Regression Analysis of AKD Based on Variables in the Nomogram

\begin{tabular}{|l|l|l|}
\hline Variables & OR $\mathbf{( 9 5 \% ~ C l )}$ & P-value \\
\hline Surgery approach, radical vs partial & $3.21(1.3 \mathrm{I}-7.88)$ & $0.01 \mathrm{I}$ \\
$\mathrm{CCl},<4 \mathrm{vs} \geq 4$ & $4.62(\mathrm{I.75-12.18)}$ & 0.002 \\
Decrement of eGFR, $\mathrm{mL} / \mathrm{min} / \mathrm{I} .73 \mathrm{~m}^{2}$ & $1.06(\mathrm{I} .04-1.08)$ & $<0.00 \mathrm{I}$ \\
\hline
\end{tabular}

Abbreviations: $\mathrm{OR}$, odds ratio; $\mathrm{Cl}$, confidence interval; $\mathrm{CCl}$, Charlson comorbidity index; eGFR, estimated glomerular filtration rate. 
Points

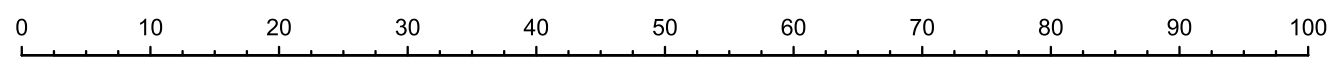

Surgery apporach

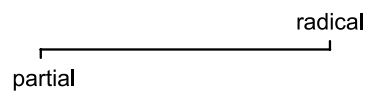

$\mathrm{CCl}$

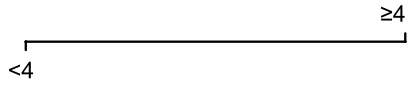

Decrement of eGFR

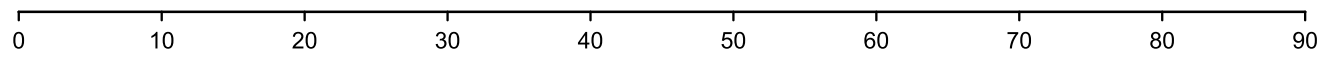

Total Points

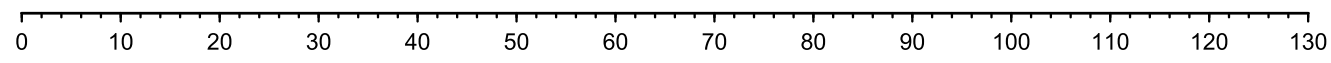

Risk of AKD

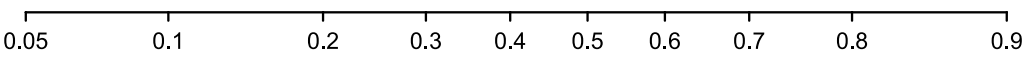

Figure I Nomogram for the prediction of AKD in patients with RCC within the 3 months after surgery, based on multivariable model. Instructions: locate the surgery approach on the corresponding axis. Draw a line straight down to the axis to calculate how many points toward the probability of AKD in the patients undergoing his/her surgery approach. Repeat the courses for $\mathrm{CCl}$ and the decrement of eGFR. Add all points obtained from the previous steps, and locate the final summation on the total score axis. The probability of the AKD corresponds to the summation score on the risk scale.

C-index of $0.76(95 \% \mathrm{CI}=0.67-0.86)$ with a good calibration curve in estimating AKD risk (Figure 2B).

Besides, we built three simple models based on factors in the nomogram model and compared the performance and clinical utility of different models. The C-index of different models in the training and validation cohorts are shown in Supplement Table 2. The area under the decision curve in Figure $3 \mathrm{~A}$ and $\mathrm{B}$ shows the clinical utility of different models. The nomogram model had a higher net benefit among a wide range of threshold probabilities than the simple models. Besides, it is better than the "treat all" (blue) or "treat none" (black) strategies in both cohorts.

\section{Discussion}

The criteria of AKD was firstly put forward in $2012 .^{11}$ The presence of AKD after surgery indicates the worsening of renal function, which is also associated with poorer prognosis. ${ }^{17}$ In 2019, a large cohort study included 1.1 million patients in Canada with $\mathrm{SCr}$ data, which suggested that the criteria of AKD identified certain group of patients with increased risks of CKD, end stage kidney disease (ESRD), and death. ${ }^{21}$ Among patients with AKD, the long-term outcomes are not predetermined and might be reversed by timely intervention, such as patient education, drug adjustment active surveillance, and frequent followup. ${ }^{13}$ Thus, early prediction of AKD and in time intervention are of vital importance. However, information on the cause of AKD after nephrectomy in RCC patients and the clinical characteristics of these patients are sparse. Previous studies have already discussed the risk factors of AKI and CKD. 5,17,19,22,23 Nonetheless, the incidence and risk factors of AKD remain unknown, and the AKD prediction model for RCC patients after nephrectomy has not been developed before. Therefore, we retrospectively collected the data of RCC patients who had undergone 
A

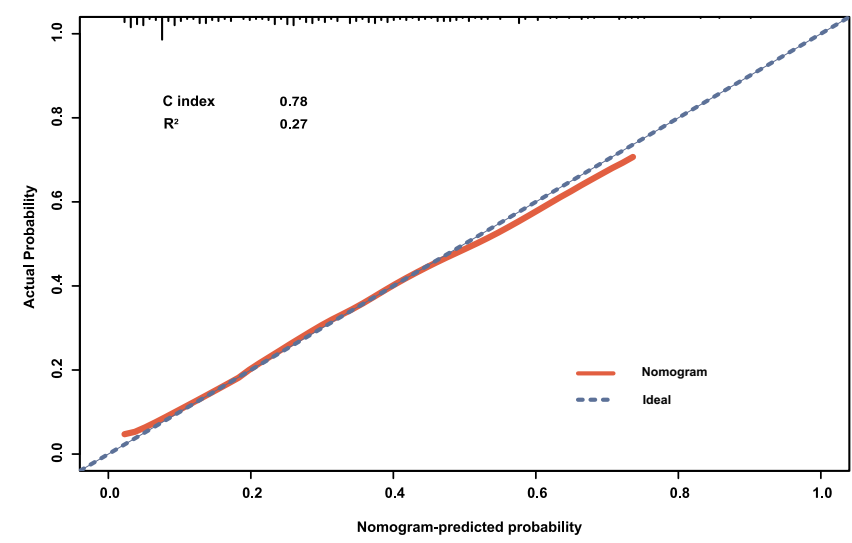

B

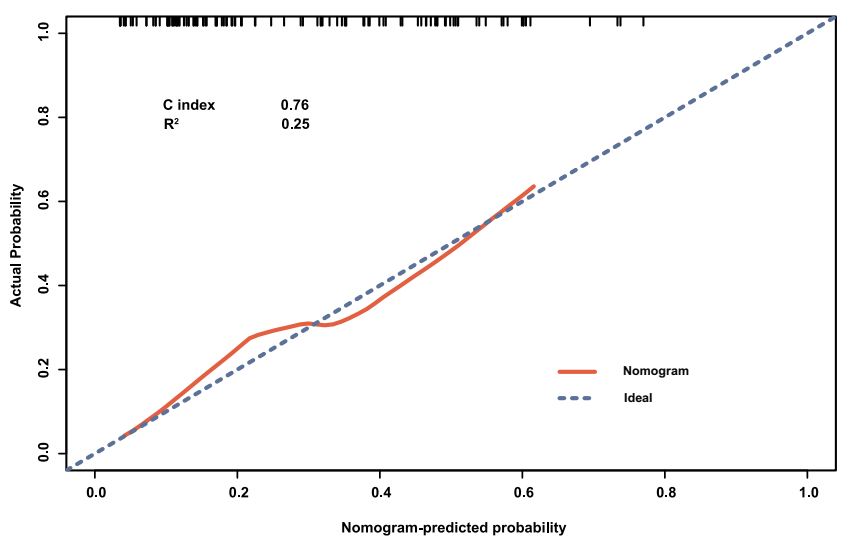

Figure 2 Calibration curve for postoperative AKD based on the nomogram. (A) training cohort, (B) validation cohort. The blue dashed line represents the ideal line of a perfect match between predicted and observed occurrence of AKD. The red line indicates the performance of the proposed nomogram.

nephrectomy in our hospital and analyzed the incidence rate and risk factors of postoperative AKD. In our study, AKD occurred in $73(27.5 \%)$ and $32(28.3 \%)$ patients in the training and validation cohorts, respectively, and we found that surgery approach, CCI, and the decrement of eGFR were independent risk factors of AKD for RCC patients after nephrectomy in multivariate analysis.

Surgery approach, which is the most efficacious treatment for RCC, is associated with a substantial risk of division on renal function. ${ }^{24}$ Previous studies reported that the rate of renal function reduction is higher in patients who had undergone radical nephrectomy than partial nephrectomy, as radical nephrectomy has a larger resection range compared to the partial nephrectomy. ${ }^{22,25,26}$ Consequently, existing guidelines recommend partial nephrectomy whenever technically feasible in an effort to maintain kidney function and optimize outcomes. $^{27}$ In a study of identifying independent predictors of AKI after radical and partial nephrectomy in RCC patients, radical nephrectomy and open surgery were associated with higher probability of postoperative AKI, which consequently affects survival. ${ }^{17,22}$ Similarly, we found that a surgery approach is an independent risk factor for AKD, which indicates the reduction of renal function after surgery. Thus, surgeons need to be more careful in choosing a surgery approach for RCC patients and reserve renal
A

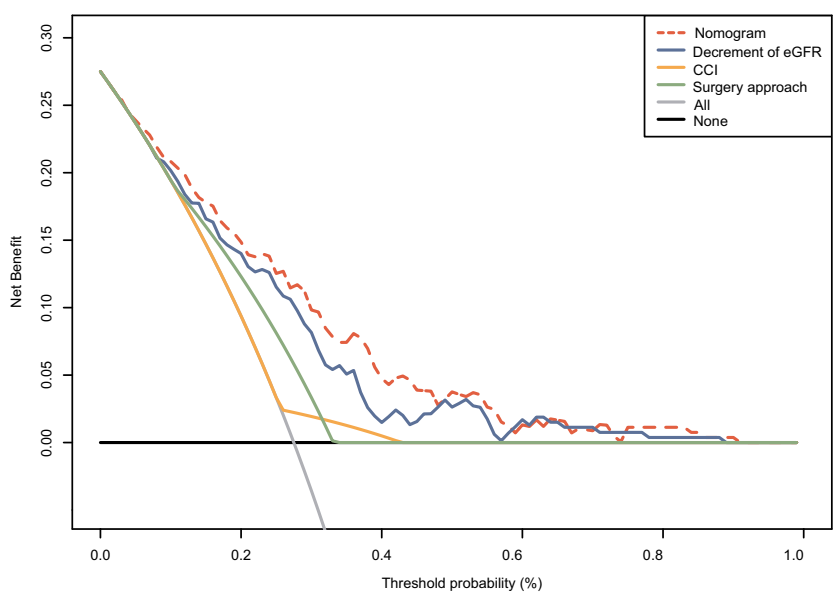

B

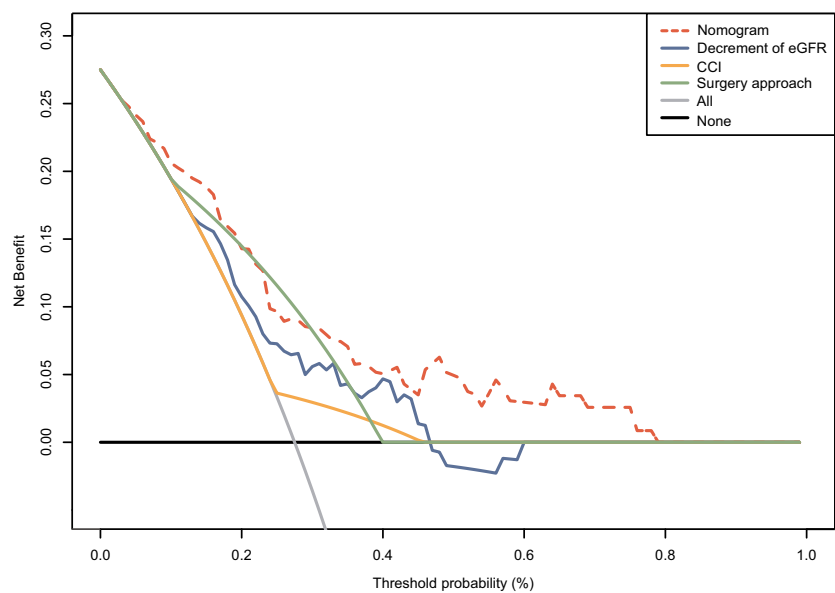

Figure 3 Decision curve of the nomogram and simple models. (A) Training cohort, (B) Validation cohort. Decision curve analyses demonstrating the net benefit associated with the use of different models for the prediction of postoperative AKD. The thick black line represents the net benefit of offering no intervention, assuming that none of the patients would develop AKD; the blue line shows the net benefit of offering interventions to all patients, assuming that all patients would develop AKD; Other lines represent the net benefit of offering interventions according to different models. 
parenchyma during surgery as excessive resection might lead to the development of AKD.

As for patients with comorbidities, previous studies showed that those with diabetes mellitus (DM) were more likely to develop CKD after nephrectomy. ${ }^{16,28,29}$ While in our study, DM alone was not significantly associated with AKD. CCI is a weighted score for each of the comorbid conditions. ${ }^{20}$ It is a comprehensive index that manifests patient's comorbidities status. Previous studies showed that CCI is a stable predictor of CKD. ${ }^{18,29}$ In our study, we found that CCI of $10.6 \%$ (28/265) and $13.3 \%$ $(15 / 113)$ patients were greater or equal to 4 in the training and validation cohort, respectively. In the multivariate analysis, CCI was independently associated with the development of AKD. In clinical practice, patients with multiple comorbidities are common. It is difficult to evaluate the effect of these comorbidities on the outcome of patients' renal function comprehensively. CCI is an easyused index and it is significantly associated with AKD, as our study demonstrated. We suggest physicians should evaluate this index for every RCC patient before surgery.

eGFR is another important indicator of kidney function after nephrectomy in RCC patients. The relationship of eGFR and CKD has been studied previously and most studies found that lower baseline eGFR independently affects the progression of CKD. ${ }^{15,30}$ Yet, in our study, baseline eGFR is not associated with the occurrence of AKD in the patients undergoing nephrectomy. The dissimilar outcomes might attribute to the different end points. In the training cohort of our study, only $20(27.4 \%)$ and two $(2.7 \%)$ patients with preoperative eGFR $60-89 \mathrm{~mL} / \mathrm{min} / 1.73 \mathrm{~m}^{2}$ and $<60 \mathrm{~mL} /$ $\mathrm{min} / 1.73 \mathrm{~m}^{2}$ had AKD, respectively. Thus, we calculated the decrement of eGFR after surgery for each patient as we think it is more relevant to the impairment of renal function caused by surgery. And, as expected, it was an independent risk factor associated with $\mathrm{AKD}$ in univariate and multivariate regression analysis. Furthermore, the decrement of eGFR was the most predictive variable for postoperative $\mathrm{AKD}$ $(\mathrm{C}$-index $=0.73)$. Thus, we suggest routine evaluation of eGFR before and after surgery for RCC patients for early detection of AKD and timely management.

After identifying independent risk factors, we built a nomogram model based on the three factors above (surgery approach, CCI, and the decrement of eGFR) in the training cohort and validate it in the validation cohort. This model demonstrated a good prediction ability with a C-index of $0.78(95 \% \quad \mathrm{CI}=0.71-0.84)$ and $0.76(95 \%$ $\mathrm{CI}=0.67-0.86)$ in the training and validation cohort, respectively. Figure $2 \mathrm{~A}$ and $\mathrm{B}$ showed that the difference between the actual and predicted values of two cohorts were small, which indicated that the model is well calibrated. The decrement of eGFR is one of the most important factors in the predicting model. Every unit decrease in eGFR indicates that the probability of developing AKD would be increased by 1.06-times for patients after surgery. Surgery and CCI also had good performance in predicting AKD alone. Therefore, we established three simple models based on the independent risk factors alone, and compared the clinical utility of the simple models with the nomogram model. In decision curve analysis (Figure 3A and B), the nomogram model had more net benefit than the simple models over a wide range of thresholds and the results are consistent in training and validation cohorts, which means the nomogram model is beneficial for clinical use.

Overall, our study firstly used the definition of AKD as the end point rather than $\mathrm{AKI}$ and $\mathrm{CKD}$, which were never studied before. We reported the incidence rate of $\mathrm{AKD}$ for RCC patients receiving nephrectomy, analyzed the risk factors of developing AKD postoperatively, and built an easy-used nomogram model to predict the probability of developing AKD for RCC patients. Proper use of this model can help physicians to identify patients with high risk of developing AKD. Close follow-up should be demanded from high risk patients, and timely intervention should be provided in order to reverse outcomes.

Our study had several limitations. Firstly, data for this study were retrospectively collected so that some patients' data were missing. For example, we did not have data on ischemia time of patients during the surgery, which was shown to affect renal function postoperatively in a prior study. ${ }^{23}$ Besides, the study was based on the retrospective observational design from our institution alone. External validation of data from other institutions could be more convincing. However, the training and validation cohorts in our study were divided according to the date of operation. Similar results from different time periods demonstrate the stability of this model to a certain extent. In addition, as a retrospective study, selection bias is inevitable as only RCC patients with complete data before and after surgery were enrolled in our study. Despite these limitations, this is the first study to establish an easy-to-use model to predict AKD in early stage after surgery. These results can provide information for clinicians to choose appropriate intervention for patients in the early postoperative period. 


\section{Conclusion}

Our study provided information on the incidence rate of AKD for RCC patients after surgery and analyzed the risk factors of developing AKD after surgery. By combining three independent risk factors of postoperative AKD, we built a nomogram model. This model can serve as an easyto-use tool for the early identification of RCC patients at high risk of developing AKD after surgery.

\section{Data Sharing Statement}

The data that support the findings of this study are available from the corresponding author upon reasonable request. The data are not publicly available due to privacy or ethical restrictions.

\section{Ethics Statement}

The study was approved by the Independent Ethics Committee of First Affiliated Hospital of Zhengzhou University (2020-KY-248). All analyses were in accordance with the Declaration of Helsinki. As the data was retrospectively collected, and no identifying information was included, the ethics committee waived the requirement for informed consent.

\section{Funding}

This work was supported by Innovation Scientists and Technicians Troop Construction Projects of Henan Province (No.182101510002) and Science and Technology Huimin Project of Henan Province (No. 162207310001).

\section{Disclosure}

The authors report no conflicts of interest for this work.

\section{References}

1. Znaor A, Lortet-Tieulent J, Laversanne M, Jemal A, Bray F. International variations and trends in renal cell carcinoma incidence and mortality. Eur Urol. 2015;67(3):519-530. doi:10.1016/j. eururo.2014.10.002

2. Ljungberg B, Campbell SC, Choi HY, et al. The epidemiology of renal cell carcinoma. Eur Urol. 2011;60(4):615-621. doi:10.1016/j. eururo.2011.06.049

3. Ljungberg B, Albiges L, Abu-Ghanem Y, et al. European association of urology guidelines on renal cell carcinoma: the 2019 update. Eur Urol. 2019;75(5):799-810. doi:10.1016/j.eururo.2019.02.011

4. Karakiewicz PI, Briganti A, Chun FK, et al. Multi-institutional validation of a new renal cancer-specific survival nomogram. J Clin Oncol. 2007;25(11):1316-1322. doi:10.1200/JCO.2006.06.1218

5. Clark MA, Shikanov S, Raman JD, et al. Chronic kidney disease before and after partial nephrectomy. $J$ Urol. 2011;185(1):43-48. doi:10.1016/j.juro.2010.09.019
6. Chung JS, Son NH, Byun SS, et al. Trends in renal function after radical nephrectomy: a multicentre analysis. BJU Int. 2014;113 (3):408-415. doi:10.1111/bju.12277

7. Hwang J, Qi L. Quality control in the endoplasmic reticulum: crosstalk between ERAD and UPR pathways. Trends Biochem Sci. 2018;43(8):593-605. doi:10.1016/j.tibs.2018.06.005

8. Clark AT, Breau RH, Morash C, Fergusson D, Doucette S, Cagiannos I. Preservation of renal function following partial or radical nephrectomy using 24-hour creatinine clearance. Eur Urol. 2008;54 (1):143-149. doi:10.1016/j.eururo.2008.03.037

9. Go AS, Chertow GM, Fan D, McCulloch CE, Hsu CY. Chronic kidney disease and the risks of death, cardiovascular events, and hospitalization. N Engl J Med. 2004;351:1296-1305. doi:10.1056/NEJMoa041031

10. Muntner P, He J, Hamm L, et al. Renal insufficiency and subsequent death resulting from cardiovascular disease in the United States. $J \mathrm{Am}$ Soc Nephrol. 2002;13(3):745-753.

11. Kellum JA, Lameire N, Aspelin P, et al. Kidney disease: improving global outcomes (KDIGO) acute kidney injury work group. KDIGO clinical practice guideline for acute kidney injury. Kidney Int Suppl. 2012;2(Suppl 1):S1-S138.

12. Levey AS, de Jong PE, Coresh J, et al. The definition, classification, and prognosis of chronic kidney disease: a KDIGO controversies conference report. Kidney Int. 2011;80(1):17-28. doi:10.1038/ki.2010.483

13. Chawla LS, Bellomo R, Bihorac A, et al. Acute kidney disease and renal recovery: consensus report of the acute disease quality initiative (ADQI) 16 workgroup. Nat Rev Nephrol. 2017;13(4):241-257. doi:10.1038/nrneph.2017.2

14. Chawla LS, Eggers PW, Star RA, Kimmel PL. Acute kidney injury and chronic kidney disease as interconnected syndromes. $N$ Engl $J$ Med. 2014;371(1):58-66. doi:10.1056/NEJMra1214243

15. Martini A, Cumarasamy S, Beksac AT, et al. A nomogram to predict significant estimated glomerular filtration rate reduction after robotic partial nephrectomy. Eur Urol. 2018;74(6):833-839. doi:10.1016/j. eururo.2018.08.037

16. Abdel Raheem A, Shin TY, Chang KD, et al. Yonsei nomogram: a predictive model of new-onset chronic kidney disease after on-clamp partial nephrectomy in patients with T1 renal tumors. Int $J$ Urol. 2018;25(7):690-697. doi:10.1111/iju.13705

17. Cho A, Lee JE, Kwon GY, et al. Post-operative acute kidney injury in patients with renal cell carcinoma is a potent risk factor for new-onset chronic kidney disease after radical nephrectomy. Nephrol Dial Transplant. 2011;26(11):3496-3501. doi:10.1093/ndt/gfr094

18. Klarenbach S, Moore RB, Chapman DW, Dong J, Braam B. Adverse renal outcomes in subjects undergoing nephrectomy for renal tumors: a population-based analysis. Eur Urol. 2011;59(3):333-339. doi:10.1016/ j.eururo.2010.11.013

19. Raman JD, Lin YK, Shariat SF, et al. Preoperative nomogram to predict the likelihood of complications after radical nephroureterectomy. BJU Int. 2017;119(2):268-275. doi:10.1111/bju.13556

20. Charlson ME, Pompei P, Ales KL, MacKenzie,CR. A new method of classifying prognostic comorbidity in longitudinal studies: development and validation. $J$ Chronic Dis. 1987;40(5):373-383. doi:10.1016/0021-9681(87)90171-8

21. James MT, Levey AS, Tonelli M, et al. Incidence and prognosis of acute kidney diseases and disorders using an integrated approach to laboratory measurements in a universal health care system. JAMA Netw Open. 2019;2(4):e191795. doi:10.1001/jamanetworkopen. 2019.1795

22. Schmid M, Abd-El-Barr AE, Gandaglia G, et al. Predictors of 30-day acute kidney injury following radical and partial nephrectomy for renal cell carcinoma. Urol Oncol. 2014;32(8):1259-1266. doi:10.1016/j. urolonc.2014.05.002

23. Martini A, Sfakianos JP, Paulucci DJ, et al. Predicting acute kidney injury after robot-assisted partial nephrectomy: implications for patient selection and postoperative management. Urol Oncol. 2019;37(7):445-451. doi:10.1016/j.urolonc.2019.04.018 
24. Rosner MH, Perazella MA. Acute kidney injury in patients with cancer. $N$ Engl J Med. 2017;376(18):1770-1781. doi:10.1056/ NEJMra1613984

25. Kim CS, Bae EH, Ma SK, Kweon SS, Kim SW. Impact of partial nephrectomy on kidney function in patients with renal cell carcinoma. BMC Nephrol. 2014;15:181. doi:10.1186/1471-2369-15-181

26. Schmid M, Krishna N, Ravi P, et al. Trends of acute kidney injury after radical or partial nephrectomy for renal cell carcinoma. Urol Oncol. 2016;34(7):293.e1-293.e10. doi:10.1016/j.urolonc.2016.02.018

27. Kim SP, Thompson RH, Boorjian SA, et al. Comparative effectiveness for survival and renal function of partial and radical nephrectomy for localized renal tumors: a systematic review and meta-analysis. J Urol. 2012;188(1):51-57. doi:10.1016/j.juro.2012. 03.006
28. Jeon HG, Jeong IG, Lee JW, Lee SE, Lee E. Prognostic factors for chronic kidney disease after curative surgery in patients with small renal tumors. Urology. 2009;74(5):1064-1068. doi:10.1016/j. urology.2009.05.090

29. Ahn T, Ellis RJ, White VM, et al. Predictors of new-onset chronic kidney disease in patients managed surgically for T1a renal cell carcinoma: an Australian population-based analysis. J Surg Oncol. 2018;117(7):1597-1610. doi:10.1002/jso.25037

30. Mukkamala A, He C, Weizer AZ, et al. Long-term renal functional outcomes of minimally invasive partial nephrectomy for renal cell carcinoma. Urol Oncol. 2014;32(8):1247-1251. doi:10.1016/j. urolonc.2014.04.012

\section{Publish your work in this journal}

Cancer Management and Research is an international, peer-reviewed open access journal focusing on cancer research and the optimal use of preventative and integrated treatment interventions to achieve improved outcomes, enhanced survival and quality of life for the cancer patient
The manuscript management system is completely online and includes a very quick and fair peer-review system, which is all easy to use. Visit http://www.dovepress.com/testimonials.php to read real quotes from published authors. 